\title{
1 Title: A paradox of cumulative culture
}

2 Running Title: Cumulative culture

3 Authors: Yutaka Kobayashi ${ }^{\text {a,b }}$, Joe Yuichiro Wakano ${ }^{c}$, Hisashi Ohtsuki ${ }^{\mathrm{d}}$

4 Affiliations: ${ }^{a}$ Research Center for Social Design Engineering, Kochi University of

5 Technology, Kochi 782-8502, Japan, ${ }^{\mathrm{b}}$ Department of Management, Kochi University of

6 Technology, Kochi 782-8502, Japan, ${ }^{c}$ Meiji Institute for Advanced Study of

7 Mathematical Sciences, Nakano, Tokyo 164-8525, Japan, ${ }^{\mathrm{d}}$ The Graduate University for

8 Advanced Studies, Shonan Village, Hayama, Kanagawa 240-0193, Japan

9 Corresponding author: Yutaka Kobayashi, Department of Management, Kochi

10 University of Technology, Kochi 782-8502, Japan, Tel +81-887-57-2344, Email

11 kobayashi.yutaka@kochi-tech.ac.jp

13 Keywords: social learning, cultural evolution, gene culture coevolution, dual inheritance

14 theory, cultural social dilemma 


\section{Abstract}

18 Culture can grow cumulatively if socially learned behaviors are improved by individual

19 learning before being passed on to the next generation. Previous authors showed that this kind of learning strategy is unlikely to be evolutionarily stable in the presence of a trade-off between learning and reproduction. This is because culture is a public good that is freely exploited by any members of the population in their model (cultural social dilemma). In this paper, we investigate the effect of vertical transmission (transmission

24 from parents to offspring), which decreases the publicness of culture, on the evolution of cumulative culture in both infinite and finite population models. In the infinite population model, we confirm that culture accumulates largely as long as transmission is purely vertical. It turns out, however, that introduction of even slight oblique transmission drastically reduces the equilibrium level of culture. Even more surprisingly, if the population size is finite, culture hardly accumulates even under purely vertical transmission. This occurs because stochastic extinction due to random genetic drift prevents a learning strategy from accumulating enough culture. Overall, our theoretical results suggest that introducing vertical transmission alone does not really help solve the cultural social dilemma problem. 


\section{$34 \quad$ 1. Introduction}

Rogers (1988) argued that the presence of culture per se does not imply improvement of population-level adaptability. This result, which contradicted the apparent advantages of culturally transmitted technologies in humans, was received with some astonishment by researchers of the day (Boyd and Richerson, 1995a). Nowadays, it is acknowledged that this "paradox" is a consequence of the specific structure of Rogers' model and can be "resolved" by taking realistic properties of human culture into account (Enquist et al. 2007; Aoki and Feldman, 2014). One of them, which may be the most relevant, is the cumulativeness of culture (Aoki et al. 2012). That is, human culture does not, as in Rogers' model, have just two states (adaptive vs. maladaptive), but evolves gradually by accumulating modifications over many generations to finally yield complex artifacts that cannot be invented by a single individual (Richerson and Boyd, 2005). It is well known that chimpanzees socially learn how to crack nuts using stones and also to fish termites using sticks (Whiten et al., 1999), but such behavior is not cumulative culture, as it fall well within the inventive capacity of a single individual. It is not comparable with spacecraft, mobile phones, and quantum mechanics, which are clearly beyond the inventive capacity of a single individual. Even basic hunter-gatherer tools like a spear are products of cumulative cultural evolution, being composed of multiple parts that 
cannot be made without some other tools like scrapers or wrenches, which may already be complex enough (Richerson and Boyd, 2005). On the other hand, ethnobotanical

54 knowledge for food-gathering and processing can be cumulative in a more quantitative sense, built upon numerous trials and errors, which can never be exerted within the lifetime of a single individual. In this view, Rogers' model is not a model of cumulative cultural evolution.

While many animal species engage in social learning and hence have culture to varying degrees (Slater, 1986; Box and Gibson, 1999; Whiten et al., 1999; Krützen et al., 2005), it is only humans that are known to have cumulative culture (Laland and Hoppitt, 2003; Tennie et al. 2009; Mesoudi, 2011a; see also Mithen, 1999). Many researchers consider that cumulative cultural evolution is a major source of adaptation in modern humans (Tomasello, 1999; Richerson and Boyd, 2004). More than two decades after Rogers' study, another paradox, which is more relevant to human evolution, has emerged. Obviously, culture can accumulate over generations only if socially learned traits undergo improvements before or while being passed on to the next generation. Such improvements can be made through deliberate individual learning (Aoki et al., 2012) or inaccurate social learning combined with success-biased transmission (Henrich, 2004). In the latter case, positive cultural growth 
is ensured in a sufficiently large, well connected population (Henrich, 2004; Powell et al., 2009; Mesoudi, 2011b; Kobayashi and Aoki, 2012). As to the former mechanism, recent models show that a learning schedule in which social learning occurs in an earlier life stage than individual learning is indeed favored by natural selection (Aoki et al. 2012). The optimal learning schedule allows culture to accumulate largely as long as improvement of traits is the sole concern of each individual. Interestingly, however, such a learning schedule loses evolutionary stability as soon as a trade-off in terms of time between learning and reproductive effort is introduced (Wakano and Miura, 2014).

It has been presumed that this occurs because of the publicness of culture; that is, a strategy that spends a lot of time to improve socially learned traits (and hence contributes to culture) allows invasion by selfish mutants that just scrounge the culture and spend the rest of time reproducing. Therefore, culture decays until finally the benefit of social learning is also lost. This results in a final state where individuals engage mainly in biological replication and little in learning (Lehmann et al. 2013; Wakano and Miura, 2014). This result contradicts the observation that modern humans possess highly cumulative, sophisticated technologies, which must have largely contributed to their current demographic success on the global scale. 
88 dilemma, where temptation to cheat prevents the population from reaching an adaptive,

89 cooperative state. They speculated that the dilemma would be overcome if cultural

90 transmission occurs mainly between close relatives, preventing cheaters from accessing

91 adaptive cultural products. For clarity, let us imagine an extreme hypothetical situation

92 where reproduction is asexual and transmission of culture is purely "vertical" (i.e. from

93 parents to their offspring (Cavalli-sforza and Feldman, 1981)). In this case, each genetic

94 lineage can be seen as an isolated population, and hence a strategy that promotes

95 accumulation of culture and is optimal from the population viewpoint should also be

96 favored by natural selection. In fact, Lehmann et al.'s (2010) model, which treats only

97 within-generation accumulation of culture, shows that culture can accumulate beyond

98 the capacity of a single individual if culture is horizontally transmitted between close

99 relatives in the same generation. However, no study tested the effect of kin transmission

100 on the evolution of between-generation cumulative culture.

101 Below, we investigate the effect of vertical transmission on the evolution of

102 between-generation cumulative culture using infinite and finite population models. Our

103 primary purpose is to test whether the privatization of culture through vertical

104 transmission can function as a theoretical mechanism to solve the above-mentioned

105 social dilemma problem. In the infinite population model, we first confirm that pure 
vertical transmission indeed solves the above-mentioned cultural social dilemma and

107 allows a large accumulation of culture. It turns out, however, that introduction of even

108 slight "oblique" transmission (i.e. transmission from a non-parental adult in the parental

109 generation (Cavalli-sforza and Feldman, 1981)) drastically reduces the equilibrium level

110 of culture. Even more surprisingly, if the population size is finite, culture hardly

111 accumulates even under pure vertical transmission. This occurs because stochastic

112 extinction of learning strategies prevents culture from accumulating enough to exert its

113 effect. In the Discussion, we will argue implications of our theoretical results for

114 empirical research.

\section{2. Methods}

117 2.1. Model description

118 We work on a simplified model to extract the essence of the problem while keeping

119 analytical tractability. In particular, we ignore the effects of environmental fluctuation,

120 which have been extensively studied by previous authors (e.g. Boyd and Richerson,

121 1985; Feldman et al., 1996; Wakano et al., 2004; Wakano and Aoki, 2006). Notation

122 used in this model is summarized in Table 1. We assume an asexually reproducing population in which generations are overlapping insofar as cultural transmission occurs. 
124 The population size is constant but may be either infinite or finite. In the finite case we

125 denote the population size by $N$.

126 Within each generation, time passes continuously; we let $\tau$ and $t$ represent the

127 generation and the within-generation time, respectively. We assume that each individual

128 in the population is distinguished from others by a unique label $i$. We may say

129 "individual $(i, \tau)$ " instead of saying "individual $i$ in generation $\tau$ " whenever it is

130 convenient. Individuals engage in three activities in a sequential manner: they first learn

131 socially, second learn individually, and finally exploit environments to reproduce. We

132 may call the first two stages collectively the learning stage. We assume this order of the

133 three activities and the discontinuous switching between activities (i.e. "bang-bang"

134 control) because they were well established in previous studies by means of dynamic

135 optimization theory (Aoki et al. 2012; Lehmann et al. 2013; Wakano and Miura, 2014).

136 It must here be noted that by the term "individual learning" we refer to an effort to add

137 to or improve knowledge or skills that an individual already possess, while "social

138 learning" refers to copying others' knowledge or skills. In this respect, we follow a

139 series of previous theoretical models (Aoki et al., 2012; Lehmann et al., 2013; Wakano

140 and Miura, 2014). We focus on the evolution of the length of time allocated to each

141 activity, which determines the extent to which culture accumulates. Each individual $(i, \tau)$ 
142 allocates fractions $v_{i, \tau}\left(1-x_{i, \tau}\right), v_{i, \tau} x_{i, \tau}$, and $1-v_{i, \tau}$ of the total lifetime $T$ to social learning,

143 individual learning, and exploitation, respectively. Without loss of generality, we set

$144 T=1$ throughout the paper; this means that we measure time in units of the lifetime of an

145 individual. Evolving parameters are $v$ and $x$, i.e. the fraction of time used for learning

146 and the ratio of the time used for individual learning to the whole learning time. We

147 assume that the strategy $(x, v)$ is coded for by a single haploid locus. In the ESS analysis

148 we assume that there are only two alleles, a wild-type allele and a mutant allele, on this

149 locus. In computer simulations, on the other hand, we allow existence of multiple alleles

150 on this locus.

151

Following previous authors, we assume that the cultural state of each

152 individual $(i, \tau)$ at within-generation time $t$ is represented by a positive real number $z_{i, \tau}(t)$

153 (e.g. Henrich, 2004; Powell et al., 2009; Aoki et al. 2012; Kobayashi and Aoki, 2012;

154 Lehmann et al. 2013; Wakano and Miura, 2014). The $z$-value $z_{i, \tau}(t)$ of an individual $(i, \tau)$

155 may represent its degree of skillfulness (e.g., in making tools), the level of

156 sophistication of knowledge (e.g. how to manufacture wild plants to extract nutrient or

157 detract toxins as efficiently as possible), or the amount of knowledge in a certain

158 category (e.g. a list of edible plants). For simplicity, we assume that $z_{i, \tau}(0)=0$ for

newborns. The $z$-value of an individual grows during its lifetime through social and 
160 individual learning. In the stage of social learning, each individual $(i, \tau)$ chooses an

161 individual in the parental generation $\tau$-1 as a role model and absorbs its knowledge. We

162 let $\left(\rho_{\tau}(i), \tau\right.$-1) denote the role model of an individual $(i, \tau)$. Zarger (2002) shows that, in a

163 Mayan farming village, the amount of ethnobotanical knowledge of a child grows

164 roughly in a decelerating, saturating manner during the age period from 4-14 through

165 social learning. In light of this, we assume that the $z$-value of individual $(i, \tau)$ grows in

166 the social learning stage as follows:

167

$168 \quad \frac{d}{d t} z_{i, \tau}(t)=\beta\left(z_{\rho_{\tau}(i), \tau-1}(T)-z_{i, \tau}(t)\right), \quad\left(0 \leq t \leq v_{i, \tau}\left(1-x_{i, \tau}\right)\right)$,

170 where $\beta$ is the efficiency of knowledge absorption. This equation allows $z_{i, \tau}(t)$ to grow

171 in a decelerating manner, conforming with the empirical data (Zarger, 2002). Note that

$172 z_{\rho_{\tau}(i),-1}(T)$ gives the $z$-value of individual $\left(\rho_{\tau}(i), \tau-1\right)$ at the end of its lifetime, which we

173 call the mature $z$-value of individual $\left(\rho_{\tau}(i), \tau-1\right)$. The role model $\left(\rho_{\tau}(i), \tau-1\right)$ is $(i, \tau)$ 's

174 parent and a random adult chosen from generation $\tau$-1 including the parent with

175 probabilities $q$ and 1- $q$, respectively. In other words, $q$ and 1- $q$ give the (backward)

176 probabilities of vertical and random oblique transmission, respectively. We ignore the

horizontal transmission in the present model to focus on between-generation 
178 accumulation of culture. This simplification is acceptable as a first step toward more

179 realistic modeling given that horizontal transmission is rare compared to vertical and

180 oblique transmission in traditional societies (Hewlett and Cavalli-Sforza, 1986;

181 Ohmagari and Berkes, 1997; Shennan and Steele, 1999; Reyes-Garcia et al., 2009).

$184 \quad \frac{d}{d t} z_{i, \tau}(t)=\alpha, \quad\left(v_{i, \tau}\left(1-x_{i, \tau}\right) \leq t \leq v_{i, \tau}\right)$

186 where $\alpha$ is the efficiency of individual learning. Throughout this paper, we set $\alpha=1$. This

187 implies that the unit of the $z$-value is the mature $z$-value that a life-long individual

188 learner could achieve.

190 while it grows at a constant rate in the individual-learning stage. This is a common

191 feature of existing learning-schedule models and is essential for the evolution of a

192 combined use of social and individual learning in a constant environment. By virtue of

193 this feature, it is beneficial to engage in social learning first, and then switch to

194 individual learning when the knowledge absorption rate in social learning drops to the same level as the efficiency of individual learning, i.e. when 
$196 \beta\left(z_{\rho_{\tau}(i), \tau-1}(T)-z_{i, \tau}(t)\right)=\alpha=1$. In the stage of exploitation, the $z$-value stays at the mature

197 value attained by the end of the learning stage, i.e.

198

$199 \quad \frac{d}{d t} z_{i, \tau}(t)=0 . \quad\left(v_{i, \tau} \leq t \leq 1=T\right)$

200

201 Note that the mature $z$-value $z_{i, \tau}(T)$ may be used as the target of social learning in the

202 next generation by the offspring of the focal individual or some other members of the

203 population. We assume that the efficiency of exploitation is proportional to this mature

$204 z$-value. In addition, we assume that the fitness of an adult is proportional to the total

205 resource income. This is a reasonable assumption, given that in humans energetic

206 income by an adult is expended not only for its own survival and reproduction but also

207 for children's survival and growth (Kaplan et al. 2000). Thus, the fitness of individual

$208(i, \tau)$ is given by

209

210

$w_{i, \tau}=z_{i, \tau}(T) \cdot\left(1-v_{i, \tau}\right) \cdot$

Fig. 1 sketches what happens in the finite-population model on the

213 between-generation time scale. We assume a so-called "Wright-Fisher"-type update for 
214 the genetic state of the population; i.e. each adult in generation $\tau$ is chosen as a parent of

215 a newborn in generation $\tau+1$ with a probability proportional to its fitness. Offspring

216 inherit their parent's strategy $(x, v)$. Thus, the genetic state of the population changes

217 from generation to generation due to natural selection and sampling drift (random

218 genetic drift). In the infinite-population model we consider the limit of the

219 finite-population model as the population size tends to infinity in such a way that

220 sampling drift disappears.

221 Although the $z$-value for newborns is $z_{i, \tau}(0)=0$ by assumption, the mature

$222 z$-value, i.e. $z_{i, \tau}(T)$ may vary even in a genetically monomorphic population. This is

223 because the mature $z$-value of an individual $(i, \tau)$ depends on the mature $z$-value of its

224 role model $\left(\rho_{\tau}(i), \tau\right.$-1), which in turn depends on the mature $z$-value of the role model's

225 role model $\left(\rho_{\tau-1}\left(\rho_{\tau}(i)\right), \tau-2\right)$, and so on. However, given that the population is genetically

226 fixed for a strategy, say $(x, v), z_{i, \tau}(T)$ reaches an equilibrium value, which is denoted by

$227 \tilde{z}(T)$. Therefore, the fitness also reaches an equilibrium value, which is denoted by $\tilde{w}$

228 (see Online Appendix A).

2.2. Aim of analysis

231 The aim of our analysis is to compare three solutions based on different optimality 
232 criteria: (i) the coordinated optimal strategy (COS), (ii) the evolutionarily stable strategy

233 (ESS) based on invasion growth rate in an infinite population model, and (iii) the ESS

234 based on fixation probability in a finite population model. Key parameters are the

235 vertical transmission rate and the population size, which have crucial effects on the

236 behavior of the model, as revealed in the result section.

238 fitness under the constraint that the population is genetically monomorphic (i.e. no

239 mutants are allowed). It does not depend on whether the population size is infinite or

240 finite. We use symbols $x^{\circ}, v^{\circ}$, and $\tilde{z}^{\circ}(T)$ to denote the COS values of $x, v$, and

$241 \tilde{z}(T)$, respectively. The COS was previously referred to as the "Pareto-optimal"

242 strategy (Wakano and Miura, 2014) but this is inappropriate given that these two

243 concepts are not always equivalent. While the COS is an ideal strategy from the

244 viewpoint of ultimate species success, there is no guarantee that it is favored by natural

245 selection. We hence derive the evolutionarily stable strategy (ESS) both for an infinite

246 population and for a finite population of size $N$ and compare it with that under the COS.

247 We use symbols $x^{*}, v^{*}$, and $\tilde{z}^{*}(T)$ to denote the ESS values of $x, v$, and $\tilde{z}(T)$, 248 respectively.

249 The COS analysis requires only that we work on the cultural dynamics in a 
genetically monomorphic population. The ESS analysis, on the other hand, requires that

251 we track both the genetic and cultural states of each individual simultaneously.

252 Specifically, we consider the fate of a mutant allele introduced into a resident population

253 which is at equilibrium with respect to the $z$-value (Fig. 1). In the case of an infinite

254 population, sampling drift is absent and the frequency of a mutant allele hence changes

255 deterministically; therefore, as in traditional analysis, we may define an ESS as a

256 strategy that does not allow any slightly deviant strategy to have a positive growth rate

257 (Maynard Smith, 1982). In the finite case, however, the frequency of a mutant allele

258 undergoes stochastic fluctuation due to sampling drift. We therefore use a definition of

259 an ESS based on a fixation probability (e.g. Nowak et al., 2004). Let $N$ be the

260 population size. We say that a strategy $\left(x^{*}, v^{*}\right)$ is evolutionarily stable if and only if the

261 fixation probability of any slightly deviated strategy in the population of the resident

262 strategy $\left(x^{*}, v^{*}\right)$ is lower than $1 / N$, i.e. the fixation probability under neutrality.

263 Unfortunately, we could not confirm analytically the second-order stability of

264 the ESS's we obtained. To confirm the evolutionary stability of the analytically derived

265 formulae and the validity of the approximations, we conducted some individual-based

266 simulations. See Online Appendices for all mathematical details. 


\section{Results}

269

3.1. Coordinated optimal strategy

270 As shown in Online Appendix A, the equilibrium fitness in a genetically monomorphic

$\tilde{w}=v(1-v) x e^{\beta v(1-x)}$.

$280 \quad v^{\circ}=\tilde{z}^{\circ}(T)=\frac{1}{2}$.

281

282 Thus, the COS involves no social learning when $\beta<2$. On the other hand, if $\beta>2$, the

283 COS involves social learning and is given by

284

$285 \quad x^{\circ}=\frac{1}{\beta-1}, \quad(6 \mathrm{c})$ 
$287 \quad v^{\circ}=1-\frac{1}{\beta}$,

$289 \quad \tilde{z}^{\circ}(T)=\frac{1}{\beta} e^{\beta-2}$.

292 learning. The absence of social learning requires $\beta<2$ for the following reason. Note that 293 from eq. (2) the absence of social learning $(x=0)$ entails $z_{i, \tau}(T)=v$. Thus, the equilibrium 294 mature $z$-value is also given by $\tilde{z}(T)=v$. Therefore, the equilibrium fitness is given by $295 \widetilde{w}=\widetilde{z}(T) \cdot(1-v)=v(1-v)$, which is maximized at $v=1 / 2$. Thus, the COS without social 296 learning, if possible, must satisfy that $v^{\circ}=\tilde{z}^{\circ}(T)=1 / 2$ in addition to $x^{\circ}=1$. However, since the COS by definition maximizes the fitness, the fitness must not increase by introducing social learning. This entails that the rate of social learning is lower than that 299 of individual learning already at birth, i.e. $\beta\left(\tilde{z}^{\circ}(T)-0\right)<\alpha=1$. Given that $\tilde{z}^{\circ}(T)=1 / 2$, this condition reduces to $\beta<2$. These arguments reveal that $\beta<2$ is a necessary condition 301 for the COS to satisfy $x^{\circ}=1$. Eq. (6) shows that the COS is solely determined by the efficiency of social learning $\beta$. It also shows that reliance on individual learning $\left(x^{\circ}\right)$ decreases with social 
304 learning efficiency $(\beta)$ while the learning time $\left(v^{\circ}\right)$ and the equilibrium mature $z$-value

$305\left(\tilde{z}^{\circ}(T)\right)$ both increase. In particular, individuals should exert maximal effort for

306 transmission of culture and minimal effort for individual learning and exploitation

$307 \quad\left(v^{\circ} \rightarrow 1, x^{\circ} \rightarrow 0\right)$ when social learning is highly efficient $(\beta \rightarrow \infty)$. The equilibrium

308 mature $z$-value $\left(\tilde{z}^{\circ}(T)\right)$ can take a huge value when social learning efficiency $(\beta)$ is

309 high (Fig. 2). This implies that a massive accumulation of culture is possible if the

310 members of a society try to maximize future fitness in a coordinated manner.

\subsection{ESS in an infinite population}

313 In Online Appendix B, we derive an Euler-Lotka characteristic equation that gives the

314 invasion growth rate of a rare mutant strategy in an infinite population. Using this equation, we can derive the ESS analytically under the assumption of small mutation

316 size (i.e. the mutant strategy is sufficiently close to the resident one). If $\beta>2$, an ESS with a positive investment in social learning $\left(x^{*}<1\right)$ exists and satisfies 
$323 \quad \tilde{z} *(T)=\frac{1}{\beta} e^{\beta v^{*}-1}$.

If $\beta<2$, the COS is also the ESS (eqs. (6a-b)). Eq. (7) shows that the ESS is unique and

326 given as an implicit function of parameters $\beta$ and $q$. When the cultural transmission is

327 purely vertical $(q=1)$, the ESS becomes equivalent to the $\operatorname{COS}\left(x^{*}=x^{\circ}, v^{*}=v^{\circ}\right)$, as

328 expected (see also Fig. 2). Close inspection of eq. (7) reveals that both learning time

$\left(v^{*}\right)$ and the equilibrium mature $z$-value $\left(\widetilde{z}^{*}(T)\right)$ are monotonically increasing and

reliance on individual learning $\left(x^{*}\right)$ is monotonically decreasing with respect to vertical

transmission probability $(q)$. Thus, the equilibrium mature $z$-value attained by the ESS is always lower than that attained by the COS.

$\left(x^{*}\right)$ are monotonically increasing and decreasing, respectively, with respect to social

learning efficiency $(\beta)$. The learning time $\left(v^{*}\right)$ is, however, not monotonic unless

efficiency $(\beta \rightarrow \infty)$ differs qualitatively between when transmission is purely vertical 
340 and the equilibrium mature $z$-value diverges $\left(v^{*} \rightarrow 1, x^{*} \rightarrow 0\right.$ and $\tilde{z}^{*}(T) \rightarrow \infty$ hold as

$341 \quad \beta \rightarrow \infty)$ (Fig. 2). If transmission is partially oblique $(q<1)$, on the other hand, we

342 obtained the following approximate formula for large $\beta$

343

$344 \quad v^{*} \approx \frac{1}{\beta} \log \frac{\beta e}{1-q},(8 \mathrm{a})$

345

$346 \quad \tilde{z}^{*}(T) \approx \frac{1}{1-q}$.

348 This suggests that, when social learning efficiency $(\beta)$ is large, introduction of rather

349 weak oblique transmission can result in a drastic fall in the equilibrium mature $z$-value.

350 For example, when $\beta=10$, the COS attains $\tilde{z}^{\circ}(T) \approx 298$, while the ESS under $q=0.99$

$351(q=0.9)$ attains only $\tilde{z}^{*}(T) \approx 24.9\left(\tilde{z}^{*}(T) \approx 4.33\right)$. This drastic reduction in the

equilibrium mature $z$-value $\left(\widetilde{z}^{*}(T)\right)$ in response to slight oblique transmission reflects a

steep reduction in the learning time $\left(v^{*}\right)$. For example, when $\beta=10$ and $q=0.99(q=0.9)$,

354 it holds that $v^{*} \approx 0.652\left(v^{*} \approx 0.477\right)$, which is much lower than $v^{\circ}=0.9$ (see also Fig. 2 ).

355 Although the ESS invests more in reproduction than the COS, this is not enough to compensate for the reduction in the mature $z$-value; that is, the ESS generally attains a 
strategy can attain a higher fitness at equilibrium than the COS in a monomorphic population. In fact, when $\beta=10$ and $q=0.99$, the ESS attains the equilibrium fitness of about $8.67\left(\approx \widetilde{z}^{*}(T) \times\left(1-v^{*}\right)=24.9 \times(1-0.652)\right)$, which is much lower than that of the $\operatorname{COS}, 29.8\left(=\tilde{z}^{\circ}(T) \times\left(1-v^{\circ}\right)=298 \times(1-0.9)\right)$. Thus, notable here is not the sign but the magnitude of the effect of the vertical transmission rate. oblique transmission may be explained as follows. Let us consider the fate of a mutant strategy that increases investment in learning compared to the resident. Although the mutant can potentially reach a higher cultural level than the resident, culture needs to accumulate for several generations to compensate for the fitness loss caused by reduced investment in reproduction. For example, if 100 generations of accumulation is necessary to compensate for the fitness loss, the compensation occurs only with probability $q^{100}$. Importantly, a single failure of vertical transmission (i.e., oblique transmission) would reset the cultural level, bringing all the increased learning efforts by ancestors to naught. This explains why the ESS and the mature $z$-value are so sensitive to the introduction of slight oblique transmission. We will give a more general (but technical) explanation in the Discussion section. 


\subsection{ESS in a finite population}

377 In Online Appendix C, we derive an approximate formula for the fixation probability of

378 a mutant strategy in a finite population of size $N$ for the special case of purely vertical transmission ( $q=1)$ using the method introduced by Rousset (2004). Using this formula,

380 we can derive the ESS for $q=1$ analytically under the assumption of small mutation size.

381 If $\beta>2$, the ESS and the equilibrium mature $z$-value $\left(\tilde{z}^{*}(T)\right)$ under purely vertical

382 transmission satisfy eqs. (6a) and (6c) plus

$384 \quad \beta\left(1-v^{*}\right)-1+\frac{1}{N}=\frac{1}{N} e^{\beta v^{*-1}}$.

If $\beta<2$, the COS is again the ESS. For partially oblique transmission $(q<1)$, we resort to individual-based simulations (see the next subsection).

size $N$ under purely vertical transmission $(q=1)$ is exactly equal to the ESS for an infinite population in which the vertical transmission rate is $q=1-1 / N$. Therefore, decreasing the population size has essentially the same effect as decreasing (increasing) the vertical (oblique) transmission rate (see Fig. 2). In particular, when social learning 
expected from the effect of vertical transmission rate $(q)$ revealed in the eq. (7a) plus the following:

Thus, the equilibrium mature $z$-value is asymptotically equal to the population size. traits that account for about 100 generations. Although one might think this result convincing, the load potentially imposed by population-size finiteness should not be underestimated. For example, when $\beta=10$, the $\operatorname{COS}$ reaches $\tilde{z}^{\circ}(T) \approx 298$ as already argued. On the other hand, the ESS under $N=100$ reaches only $\tilde{z} *(T) \approx 24.9$. Moreover, in reality there would be some oblique transmission, which should further drastically

409 reduce the equilibrium mature $z$-value. In the next subsection, this effect is explored by means of computer simulations. 
412 cultural level because it creates room for stochastic extinction of rare alleles. As

413 mentioned in the previous subsection, a mutant strategy that invests more in learning

414 than the resident must endure for several generations before culture accumulates enough

415 to compensate for the fitness loss caused by decreased investment in reproduction. In

416 other words, such mutant strategy is far-sighted compared to the resident, investing in

417 the future cultural quality at the expense of present reproduction. If the population size

418 is infinite and transmission is purely vertical, this may be a good strategy; although the

419 mutant population would initially decrease, it may eventually start increasing after

420 culture enough accumulates. In a finite population, however, the mutant strategy is

421 highly likely to go extinct in the initial stage where the mutant still has lower fitness

422 than the resident. For this reason, near-sighted strategies (i.e. large investment in

423 reproduction) tend to be favored over far-sighted ones (i.e. large investment in learning)

424 in a small population. We will provide a more detailed explanation in the Discussion.

\subsection{Individual-based simulations}

427 In the simulations we explicitly tracked the changes in both genetic and cultural states of each of $N$ individuals. We assumed that each of traits $x_{i, \tau}$ and $v_{i, \tau}$ of each individual

429 can independently mutate in every generation with the same probability $\mu=0.001$. If 
mutation occurred to a trait, the new trait value was sampled from a Gaussian

431 distribution centered around the original trait value with variance $\sigma^{2}=0.001$. If the

432 sampled value turns out to be outside a boundary ( 0 or 1$)$, the new trait value was set to

433 the boundary value. As a result of recurrent mutation, many different strategies coexist

434 at each snapshot, whereas in the analytical theory we assumed there were at most only

435 two strategies (the mutant and the resident). All the other assumptions were unchanged

436 from the description in section 2.

437

We first checked if the ESS for purely vertical transmission $(q=1)$ predicted by

438 eqs. (7a), (7c), and (9) is attained in individual-based simulations. Fig. 3 shows a typical

439 time-series behavior of the population-averages of $x_{i, \tau}, v_{i, \tau}$, and $z_{i, \tau}(T)$, which are denoted

440 by $\bar{x}, \bar{v}$, and $\bar{z}(T)$, respectively. Clearly, these values all converge to the analytical

441 ESS values (broken bold lines). In the simulation of Fig. 3, the initial trait values are set

442 to the $\operatorname{COS}$; i.e. $\beta=10, v_{i, \tau}=v^{\circ}=0.9, x_{i, \tau}=x^{\circ}=0.1$ (see eqs. $(6 \mathrm{c})$ and $(6 \mathrm{~d})$ ). The role model's

$443 z$-value in the first generation was set to zero for all individuals. Thus, if there were no

444 genetic evolution, the average mature $z$-value $\bar{z}(T)$ should increase to $\tilde{z}^{\circ}(T) \approx 298$

445 according to eq. (6e). In fact, as Fig. 3b shows, $\bar{z}(T)$ initially increases up to about

$446 \tilde{z}^{\circ}(T)$ but subsequently decreases to $\tilde{z}^{*}(T)$ following the evolutionary changes in

$447 \bar{x}$ and $\bar{v}$. 
449 The figure again shows that in general the analytical theory accurately predicts

450 simulation results under purely vertical transmission except the equilibrium values of $451 \bar{z}(T)$ for some large $\beta$ (Fig. 4c). This deviation occurred because the value of $\bar{z}(T)$

452 fluctuates a lot when $\beta$ is large. As expected from the result of the infinite-population model (Fig. 2), $\bar{x}$ is not sensitive to change in $q$ (Fig. 4a). On the other hand, $\bar{v}$

454 significantly decreases with decreasing $q$ (Fig. 4b) and, as a result, $\bar{z}(T)$ sharply 455 decreases (Fig. 4c).

\section{Discussion}

\subsection{Summary of results}

Wakano and Miura (2014) argued that the public nature of culture prevents the

evolution of between-generation cumulative culture. They proposed kin selection as a mechanism to avoid this cultural social dilemma problem. We have confirmed that in our simple infinite-population model cumulative culture can evolve if social transmission is purely vertical and hence the relatedness between the donor and the recipient of information is unity $(R=1)$. However, as soon as a small probability of oblique transmission is introduced, the equilibrium level of culture drastically reduces. 
Moreover, by analyzing a model of finite population, we have shown that the vertical transmission.

\subsection{Effect of oblique transmission}

These surprising results illuminate another (i.e. other than being public) pitfall of between-generation cumulative culture, which was previously not perceived. Namely, it takes a number of generations before culture accumulates enough to compensate for the fitness loss caused by an increased investment in learning. Therefore, a mutant strategy that increases investment in learning compared to the resident must accumulate culture vertically for a number of generations without interruption by oblique transmission before it can enjoy increased fitness. Thus, the crucial determinant for the success of the mutant is the expected number of generations until a sequence of vertical transmission is terminated by oblique transmission, which is given by the reciprocal of the oblique transmission rate, i.e. $1 /(1-q)$. This quantity is obviously very sensitive to $q$ when $q$ is close to unity and reduces to a very small value as soon as $q$ gets away from unity. Interestingly, the equilibrium mature $z$-value under the ESS is also given by the reciprocal of the oblique transmission $1 /(1-q)$ when $\beta$ is very large (eq. (8b)). These 
arguments reveal why the ESS and its equilibrium mature $z$-value are both very

486 investigated the effects of transmission modes on cultural evolution (e.g., Cavalli-Sforza

488 Kobayashi and Aoki, 2012), but we have first investigated the effects of transmission accumulation of culture from the viewpoint of kin selection and the cultural social dilemma.

\subsection{Effect of population size}

494 On the other hand, it may be more difficult to understand the large effect of population size on the evolution of cumulative culture, which is evident even under pure vertical transmission. To understand this effect, let us consider why a mutant with the COS cannot be successful in the population of the ESS. Suppose that the transmission is purely vertical and the COS is initially expressed by a single mutant individual. Since

499 the COS invests less in reproduction than the ESS, the fitness of mutants is lower than 500 residents in early generations. However, it gradually increases because of the cumulative effect of culture, eventually exceeding the resident fitness (Fig. 5). 
502 Therefore, if the population size were infinite, mutants should first decrease but

503 eventually start increasing, finally reaching fixation. In a finite population, however,

504 mutants are highly likely to go extinct in the initial phase of reduced fitness before they

505 can enjoy increased fitness (see Fig. 5). This is why the COS cannot invade the ESS in a

506 finite population. Likewise, it is easy to show that the COS cannot resist against

507 invasion by the ESS in a finite population.

508 These arguments are consistent with the result of Lehmann et al. (2010), who

509 showed that culture can accumulate beyond the capacity of a single individual within a

510 generation if horizontal transmission of culture occurs mainly between genetically

511 related individuals, so that culture is essentially private. In their model, fitness reduction

512 of an elaborate learner due to decreased time for reproduction is immediately

513 compensated by beneficial information horizontally transmitted from its relatives. Thus,

514 the delay effect revealed in our model is absent in their model of within-generation

515 cumulative culture. Further arguments about this subject are given in section 4.5.

518 In the current model, we assumed that each individual engages in social learning,

519 individual learning, and exploitation of the environment in this order. Although this 
520 assumption is based on the results of previous theoretical models, it would obviously be

521 desirable to have some empirical evidence to support it. As to the assumption that

522 learning occurs in an earlier stage than exploitation of the environment, it is known that

523 in hunter-gatherer societies the energetic income by an individual during the childhood

524 is typically negligible or very small but shows a steep increase from the adolescence to

525 the early adulthood (Kaplan et al., 2000). On the other hand, most subsistence

526 knowledge and skills are mastered by the early adulthood (e.g. Ohmagari and Berkes,

527 1997; Zarger, 2002). Thus, our assumption that the learning stage precedes the

528 exploitation stage may be acceptable (though learning often requires children to

529 accompany adults on subsistence work for observation and hands-on practices, see e.g.

530 Ohmagari and Berkes, 1997).

531 Unfortunately, there is little empirical support for the assumption that

532 individual learning occurs in a later stage of life than social learning. It is relatively well

533 understood how social learning proceeds in the lifespan of an individual; for example,

534 Zarger (2002) reports that children's ethnobotanical knowledge (names and use of

535 plants) grows rapidly during the age period of 4-7 years and then at a lower rate until

536 finally it reaches the adult level during the age period of 10-14 years. On the other hand,

537 it is largely unknown how and when individual learning takes place. 
539 learning in the learning stage is not crucial to our analysis. In fact, even if each

540 individual engages in individual learning with probability $x$ and in social learning with

541 probability 1-x at any moment in the learning stage, we can reach the same conclusion.

542 To see this, let us interpret the skill level $z_{i, \tau}(t)$ specifically as the amount of (e.g.

543 ethnobotanical) knowledge individual $(i, \tau)$ has obtained through individual and social

544 learning by time $t$. In addition, assume that the knowledge produced by individual

545 learning does not overlap with that obtained by social learning. Then, as revealed in

546 Online Appendix D, the final amount of knowledge (or the skill level) obtained by the

547 end of the learning stage is given by exactly the same equation as in the original model.

548 Thus, our results do not necessarily depend on the sequential occurrence of social and

549 individual learning.

550

\subsection{Stacking versus gathering}

Perhaps it would be useful to conceptualize two kinds of cultural accumulation, which

are on the two extremes of a continuum. The first is accumulation in a horizontal sense.

In this type of accumulation, each individual reaches a high skill level by gathering 
556 inherits little culture from earlier generations. The second is accumulation in a vertical

557 sense. In this type, each individual reaches a high skill level by stacking the wisdom of

558 ancestors. There is little communication between different lines of stacks except for

559 sharing common cultural ancestors at certain points in the past. Lehmann et al. (2010)

560 suggest that the former type of accumulation is favored by natural selection, while our

561 study suggests that the latter is not. It is largely unknown to what extent intermediate

562 types of accumulation are favored by natural selection. Further theoretical research is

563 demanded.

It is worth noting that horizontal transmission per se does not generate

information inflow into a generation from outside. It just allows individuals of the same generation to exchange skills and knowledge, decreasing the variation between them

567 (Cavalli-Sforza and Feldman, 1981). On the other hand, between-generation transmission allows information inflow into a generation from past generations. Our naïve intuition tells us that modern technologies are built upon a stack of knowledge accumulated over centuries or even millennia. However, the cultural social dilemma in this type of cumulative cultural evolution (i.e., the vertical sort of accumulation) turned out to be very difficult to avoid, at least by means of privatization of culture, compared to the same problem in the horizontal sort of knowledge accumulation. 
576 Empirical data from traditional societies apparently show that knowledge and skills are

577 mostly transmitted vertically or obliquely, and rarely horizontally between peers of

578 similar ages (Hewlett and Cavalli-Sforza, 1986; Ohmagari and Berkes, 1997; Shennan

579 and Steele, 1999; Reyes-Garcia et al., 2009). For example, according to Hewlett and

580 Cavalli-Sforza (1986), the vertical transmission rates of various skills in Aka pygmies,

581 depending on skill categories, range from $q=0.519$ (for singing skills) to $q=0.893$ (for

582 food acquisition skills) and is on average $q=0.807$. Reyes-Garcia et al. (2009), analyzing

583 the relative contributions of vertical, oblique, and horizontal transmission for

584 ethnobotanical knowledge in Tsimane', an Amerindian gatherer-horticulturalist society,

585 concluded that contribution of oblique transmission dominates over that of vertical

586 transmission, suggesting that $q<0.5$. Eq. (8b) shows that the ESS mature $z$-value under

$587 q=0.5$ never exceeds 2 . The exact value of the ESS mature $z$-value depends on the

588 efficiency of social learning $\beta$. If $10 \%$ of the lifetime is required to learn a half of the

589 role model's knowledge, $(\beta \approx 6.93)$, the ESS mature skill level is $\tilde{z}^{*}(T) \approx 1$, which

590 equals the level that an individual would attain if he/she spends $100 \%$ of his/her lifetime

591 in individual learning. On the other hand, the corresponding value for the COS under 
592 the same value of $\beta$ is $\tilde{z}^{\circ}(T) \approx 20$. Thus, in light of empirical data on vertical

593 transmission rates, our model suggests that the privatization of culture by vertical

594 transmission cannot provide a satisfactory explanation for the avoidance of the cultural

595 social dilemma problem in human societies.

596 Given that vertical transmission is not a promising mechanism to avoid the

597 cultural social dilemma, we may hypothesize that culture is actually accumulating

598 mainly in a horizontal fashion (see section 4.5). This hypothesis, however, again seems

599 contradict data; i.e., horizontal transmission rates between peers in empirical data

600 usually appear to be too low to explain cumulative culture (Hewlett and Cavalli-Sforza,

601 1986; Ohmagari and Berkes, 1997; Reyes-Garcia et al., 2009). For example,

602 Reyes-Garcia et al. "did not find any evidence of horizontal transmission of

603 ethnobotanical knowledge" in the Tsimane' (Reyes-Garcia et al., 2009). Shennan and

604 Steele (1999), summarizing a range of ethnographic information concerning cultural

605 transmission of craft skills, found that vertical transmission is the dominant mode in

606 most cases and horizontal transmission is in contrast very rare with few exceptions. If

607 culture is mostly transmitted between, not within, generations as suggested by data, how

608 can the cultural social dilemma problem be solved?

609 One possibility is that horizontal transmission rate is "effectively" much higher 
610 than it appears to be (Zarger, 2002). Claidière and Andrè (2012) point out, reviewing a

611 considerable number of studies, that the mode of cultural transmission typically depends

612 (not only on the categories but also) on the contents of information transmitted. In

613 particular, data suggest that novel skills are much more likely to be transmitted

614 horizontally than well-established skills (Claidière and Andrè, 2012). Indeed, the skill

615 for making a crossbow, which is relatively new, spread exceptionally through horizontal

616 transmission in Aka pygmies (Hewlett and Cavalli-Sforza, 1986). This transmission

617 mode is not exactly the same as the success-biased transmission mode typically

618 assumed in mathematical models. In models, it is usually assumed that a social learner

619 simply chooses the best among several potential role models (Boyd and Richerson,

620 1985; Henrich, 2004). However, in the transmission mode under consideration, a social

621 learner chooses a "default" role model (often the same-sex parent) unless he/she finds a

622 remarkably successful role model. More empirical studies are demanded to confirm the

623 ubiquity of such content-dependent switching between a default and temporary

624 transmission mode.

625

626

4.7. Lucky mistakes versus deliberate inventions

627 It is worth noting again that in our terminology the term "individual learning" refers to 
628 deliberate effort to add to or improve knowledge that one already bears, as opposed to

629 absorbing knowledge that others bear. So far, all of our arguments stand upon the

630 premise that such individual learning is a prerequisite for cumulative cultural evolution.

631 We remark that this is not a widely accepted view. As briefly mentioned in the

632 introduction, theory predicts that success-biased transmission can drive cumulative

633 cultural evolution by filtering errors made in the process of social learning and selecting

634 only adaptive ones (Enquist and Ghirlanda, 2007; Henrich, 2004). This mechanism is

635 free from the cultural social dilemma problem, and therefore can be an alternative

636 mechanism to explain observed cumulative culture in human societies. We remark that

637 the essential difference between deliberate individual learning and copy error is whether

638 it is a genetically heritable strategy or not. Heritable propensities toward increased

639 errors, if any, would respond to natural selection, and therefore would suffer from a

640 similar problem to that found in our model.

641

A relevant question would be then what are the relative contributions of

642 deliberate individual learning versus copy errors in cumulative cultural evolution.

643 Unfortunately, we do not know of any empirical data to answer this question. Although

644 complex artifacts or knowledge such as spacecraft or quantum mechanics is probably

645 not a mere collection of successful copy errors accumulated by scientists for decades, 
646 we have to be careful in claiming this because Darwinian evolutionary processes, which

647 are driven by copy errors filtered by natural selection, can shape much more

648 complicated and well-functioning machineries than ever been made by humans

649 (Dawkins, 1976). Empirical studies are strongly demanded to elucidate the relative

650 contributions of individual learning and copy errors to human cumulative culture.

651

652 4.8. Other hypotheses and remarks

653 To be fair, we remark that the above-mentioned inconsistency between theory and data

654 might possibly be an artifact. In particular, as previous authors already pointed out,

655 learning and exploitation may not always be mutually exclusive (Wakano and Miura,

656 2014). In reality, experiences gained through exploitation of environments may promote

657 learning, for example, by increasing the probability of success in trial and error. It is

658 also possible that our models correctly describe real human evolution and the paradox

659 does not exist in this sense. This view entails that our culture is the result of the

660 competition among selfish individuals (i.e., ESS), and our modern technologies can,

661 contrary to our intuition, be accounted for by a few generations of individual learning.

662 This in turn implies that, if we had tried our best in a more coordinated manner (i.e.,

663 COS), we might have achieved much higher technology; e.g., we might have already 
664

665

666

667

668

669

colonized another solar system.

Finally, yet another interesting interpretation of our counterintuitive result is

that it is the very reason why cumulative culture is so rare in the biological world while

culture itself is not (Boyd and Richerson, 1995b). That is, cumulative culture is in

general disfavored, but only modern humans may have solved this problem. Then, how

did they solve the problem? One possible answer is the cooperative nature of humans.

There is evidence from paleoanthropology that evolution of human cooperativeness

preceded emergence of behavioral modernity, suggesting that the former was a

prerequisite for the latter (Dubreuil, 2010). For example, if role models are paid back

from novices in some way, motivation to learn might overwhelm temptation to take a

free ride on culture, promoting the evolution of cumulative culture. However, recent

experiments suggest that information access costs may inhibit the evolution of

cumulative culture by reducing the benefit of social learning (Mesoudi, 2008). On the

other hand, another experiment shows the price of information depends on whether

sellers and buyers share a common interest (Derex et al., 2014). Clearly, in future work

we need to combine economic and evolutionary modeling to see how information

trading within cooperatively breeding groups affects the evolution of cumulative

culture. 
683 role in the evolution of cumulative culture in modern humans (see eq. (8b)). Some

684 researchers hypothesize that population size was a crucial determinant of the Upper

685 Paleolithic revolution and the subsequent demographic expansion of modern humans

686 (Powell et al., 2009). This hypothesis is based on the theoretical prediction that a larger

687 population has a higher chance of yielding successful transmission errors, which can

688 subsequently spread over the population through success-biased social learning

689 (Henrich, 2004). The significance of population size on cumulative cultural evolution

690 has also been confirmed by experiments (Derex et al. 2013; Kempe and Mesoudi, 2014).

691 Our result supports the importance of demography, although the underlying mechanism

692 is quite different from those proposed in previous studies.

693

694

695 Acknowledgments. We thank MW Feldman, the editor, and three anonymous reviewers

696 for valuable comments. This work is supported by Monbukagakusho grant 22101004 to

697 JYW and YK and Monbukagakusho grant 25101704 and 25118006 to HO.

698

699 


\section{Literature Cited}

701

702 Aoki, K., Feldman, M. W. 2014. Evolution of learning strategies in temporally and

Aoki, K., Lehmann, L., Feldman, M. W., 2011. Rates of cultural change and patterns of cultural accumulation in stochastic models of social transmission. Theor. Popul. Biol. 79, 192-202.

708

Aoki, K., Wakano, J. Y., Lehmann, L. 2012. Evolutionarily stable learning schedules and cumulative culture in discrete generation models. Theor. Popul. Biol. 81, 300-309. Press, Cambridge.

714 Boyd, R., Richerson, P. J. 1985. Culture and the evolutionary process. The University of

715 Chicago Press, Chicago.

Boyd, R., Richerson, P. J. 1995a. Why does culture increase human adaptability? Ethol. 
718 Sciobiol. 16, 125-143.

719

720 Boyd, R., Richerson, P. J. 1995b. Why culture is common, but cultural evolution is rare.

721 Proc. Brit. Acad. 88, 73-93.

722

723 Cavalli-sforza, L. L., Feldman, M. W. 1981. Cultural transmission and evolution: A

724 quantitative approach. Princeton University Press, Princeton.

725

726 Claidière, N., Andrè, J.-B., 2012. The transmission of genes and culture: a questionable 727 analogy. Evol. Biol. 39, 12-24.

728

729 Dawkins, R. 1976. The selfish gene. Oxford University Press, Oxford.

730

731 Derex, M., Beugin, M.-P. Godelle, B., Raymond, M. 2013. Experimental evidence for

732 the influence of group size on cultural complexity. Nature 503, 389-391.

733

734 Derex, M., Godelle, B., Raymond, M. 2014. How does competition affect the

transmission of information? Evol. Hum. Behav. 35, 89-95. 
737 Dubreuil, B. 2010. Paleolithic public goods games: why human culture and cooperation

738 did not evolve in one step. Biol. Philos. 25, 53-73.

739

740 Enquist, M., Eriksson, K., Chirlanda, S. 2007. Critical social learning: A solution to

741 Rogers' paradox of nonadaptive culture. Am. Anthropol. 109, 727-734.

742

743 Enquist, M., Strimling, P., Eriksson, K., Laland, K., Sjostrand, J., 2010. One cultural

744 parent makes no culture. Anim. Behav. 79, 1353-1362.

745

746 Feldman, M. W., Aoki, K., Kumm, J. 1996. Individual versus social learning:

747 Evolutionary analysis in a fluctuating environment. Anthropol. Sci. 104: 209-232.

748

749 Enquist, M., Ghirlanda, S., 2007. Evolution of social learning does not explain the

750 origin of human cumulative culture. J. Theor. Biol. 246, 129-135.

751

752 Henrich, J. 2004. Demography and cultural evolution: how adaptive cultural processes

753 can produce maladaptive losses - the Tasmanian case. Am. Antiquity 64, 197-214. 
755 Hewlett, B. S., Cavalli-Sforza, L. L., 1986. Cultural transmission among Aka pygmies.

756 Am. Anthropol. 88, 922-934.

757

758 Kaplan H., Hill, K., Lancaster, J. Hurtado, A. M., 2000. A theory of human life history

evolution: diet, intelligence, and longevity. Evol. Anthropol. 9, 156-185.

760

761

Kobayashi, Y., Aoki, K., 2012. Innovativeness, population size, and cumulative cultural 762 evolution. Theor. Popul. Biol. 82, 38-47.

763

764 Krützen, M., Mann, J., Heithaus, M. R., Connor, R. C., Bejder, L., Sherwin, W. B., 2005.

765 Cultural transmission of tool use in bottlenose dolphins. Proc. Natl. Acad. Sci. USA 102, 8939-8943.

767

768

Laland, K. N., Hoppitt, W. 2003. Do animals have culture? Evol. Anthropol. 12,

769 150-159.

770

771 Lehmann, L., Feldman, M. W., and Kaeuffer, R. 2010. Cumulative cultural dynamics 
772 and the coevolution of cultural innovation and transmission: an ESS model for

773 panmictic and structured populations. J. Evol. Biol. 23, 2356-69.

774

775

Lehmann, L., Wakano, J. Y., and Aoki, K. 2013. On optimal learning schedules and the marginal value of cumulative cultural evolution. Evolution, 67, 1435-1445.

777

778

Maynard Smith, J. 1982. Evolution and the Theory of Games. Cambridge University

779

Press.

780

781

Mesoudi, A. 2008. An experimental simulation of the "copy-successful-individuals"

782

cultural learning strategy: adaptive landscapes, producer-scrounger dynamics, and

informational access costs. Evol. Hum. Behav. 29, 350-363.

784

Mesoudi, A., 2011a. Cultural evolution. The University of Chicago Press, Chicago.

785

786

Mesoudi, A., 2011b. Variable cultural acquisition costs constrain cumulative cultural

787

evolution. PLoS ONE, 6(3), e18239. doi:10.1371/journal.pone.0018239

788

Nowak, M. A., Sasaki, A., Taylor, C. Fundenberg, D. 2004. Emergence of cooperation 
and evolutionary stability in finite populations. Nature 428: 646-650.

791

792 Ohmagari, K., Berkes, F., 1997. Transmission of indigenous knowledge and bush skills

793 among the western James Bay Cree women of subarctic Canada. Hum. Ecol. 25,

$794 \quad 197-222$.

795

796 Powell, A., Shennan, S., Thomas, M. G. 2009. Late Pleistocene demography and the

appearance of modern human behavior. Science 324: 1298-1301.

798

799

Reyes-Garcia, V., Broesch, J., Calvet-Mir, L., Fuentes-Peláez, N., McDade, T. W., Parsa,

800

S., Tanner, S., Huanca, T., Leonard, W. R., Martínez-Rodríguez, M. R., 2009. Cultural

801

transmission of ethnobotanical knowledge and skills: an empirical analysis from an

802 Amerindian society. Evol. Hum. Behav. 30, 274-285.

803

804 Richerson, P. J., Boyd, R. 2004. Not by genes alone. The University of Chicago Press,

805 Chicago.

806

807 Rogers, A. R. 1988. Does biology constrain culture? Am. Anthropol. 90, 819-831. 
809 Shennan, S. J., Steele, J., 1999. Cultural learning in hominids: a behavioural ecological

810 approach, in: Box, H. O., Gibson, K. R. (Eds.), Mammalian Social Learning. Cambridge

811 University Press, Cambridge, pp. 367-399.

812

813 Slater, P. J. B., 1986. The cultural transmission of bird song. Trends Ecol. Evol. 1,

$81494-97$.

815

816 Tennie, C., Call, J., Tomasello, M. 2009. Ratcheting up the ratchet: on the evolution of

817 cumulative culture. Philos. Trans. R. Soc. B. 2009, 364, 2405-2415.

818

819 Tomasello, M. The cultural origins of human cognition, Harvard University Press.

820

821 Wakano, J. Y., Aoki., K. 2006. A mixed strategy model for the emergence and

822 intensification of social learning in a periodically changing natural environment. Theor.

823 Popul. Biol. 70:486-497.

824

825 Wakano, J. Y., Aoki, K., Feldman, M. W. 2004. Evolution of social learning: a 
826 mathematical analysis. Theor. Popul. Biol. 66:249-258.

827

828 Wakano, J. Y., Miura, C. 2014. Trade-off between learning and exploitation: The

829 Pareto-optimal versus evolutionarily stable learning schedule in cumulative cultural

830 evolution. Theor. Popul. Biol. 91, 37-43.

831

832 Whiten, A., Goodall, J., McGrew, W. C., Nishida, T., Reynolds, V., Sugiyama, Y., Tutin,

833 C. E. G., Wrangham, R W., Boesch. C. 1999. Cultures in chimpanzees. Nature 399,

$834 \quad 682-685$.

835

836 Zarger, R. K., 2002. Acquisition and transmission of subsistence knowledge by Q'eqchi'

837 Maya in Belize, in: Stepp, J. R., Wyndham, F. S., Zarger, R. K. (Eds.), Ethnobiology and

838 Biocultural Diversity. University of Georgia Press, Athens, pp. 593-603.

839

840 
843 Fig. 1: A schematic diagram to illustrate the between-generation processes in the

844 finite-population model. Circles and squares in each row represent resident and mutant

845 individuals, respectively, in a specific generation, where the generation number

846 increases downward. Solid and dotted arrows represent genetic and cultural

847 transmission, respectively. Simultaneous occurrence of genetic and vertical cultural

848 transmission is represented by a two-headed solid arrow. Each individual $(i, \tau)$ is given

849 two numbers, the lower one of which represents the mature $z$-value $z_{i, \tau}(T)$ of the

850 individual and the upper one the mature $z$-value of the role model $z_{\rho_{\tau}(i), \tau-1}(T)$ (for

851 simplicity we omitted the subscript of $\rho$ ). The values of $z_{i, \tau}(T)$ and $z_{\rho_{\tau}(i), \tau-1}(T)$ in the

852 diagram are not precise values obtained from theory but are artificial values for

853 illustration. In generation 1, a single mutant is introduced in a resident population of

854 size 5 which is at cultural equilibrium (the star represents the point where mutation

855 occurred). The equilibrium $z$-value of the resident strategy is set as 5. It is assumed that

856 the mutant strategy invests more time in individual and/or social learning than the

857 resident, so that mutants tend to increase the mature $z$-value. The diagram shows a case

where mutants go extinct in generation 4 . Such extinction can be caused either by i) the 
859 decrease in mutant's fitness due to increased learning time or ii) by random genetic drift even if mutant strategy has selective advantage.

861

862 Fig. 2: Effect of $\beta$ on (a) $x^{*}$, (b) $v^{*}$, and (c) $\tilde{z}^{*}(T)$ for different values of $q$ in the

863 infinite population model. The thick solid lines represent the COS (i.e., $x^{\circ}, v^{\circ}$, and

$\left.864 \tilde{z}^{\circ}(T)\right)$. The same graphs also represent the results for different population sizes in the

865 finite population model with pure vertical transmission (corresponding population sizes

866 are shown in parentheses). Note that the infinite population model and the finite

867 population model with pure vertical transmission yield equivalent results when $q=1-1 / N$.

869 Fig. 3: A typical time-series behavior of (a) $\bar{x}, \bar{v}$, and (b) $\bar{z}(T)$ (all averaged over 870 the population) obtained in the individual-based simulations. Parameter values are $\beta=10$,

$871 q=1.0$, and $N=100$. The thick dotted lines represent equilibrium values predicted by the analytical theory. The initial condition is $x=0.1$ and $v=0.9$ for all individuals.

874 Fig. 4: The effect of $\beta$ on the equilibrium values of (a) $\bar{x}$, (b) $\bar{v}$, and (c) $\bar{z}(T)$ (all averaged over the population) for three values of $q(1.0,0.99$, and 0.9$)$. Population size is $N=100$. The equilibrium values are computed by running a simulation for $10^{7}$ 
877 generations and then averaging the values over the last $5 \times 10^{6}$ generations.

878

879 Fig. 5: The dynamics of the mutant (thin solid line) and resident (thick solid line)

880 fitnesses in the finite-population model when the mutant strategy is the COS and the

881 resident strategy is the ESS. The dotted line represents the probability that mutants do

882 not go extinct before a given generation under selective neutrality. Parameter values are

$883 \beta=10, N=100$, and $q=1.0$.

884 


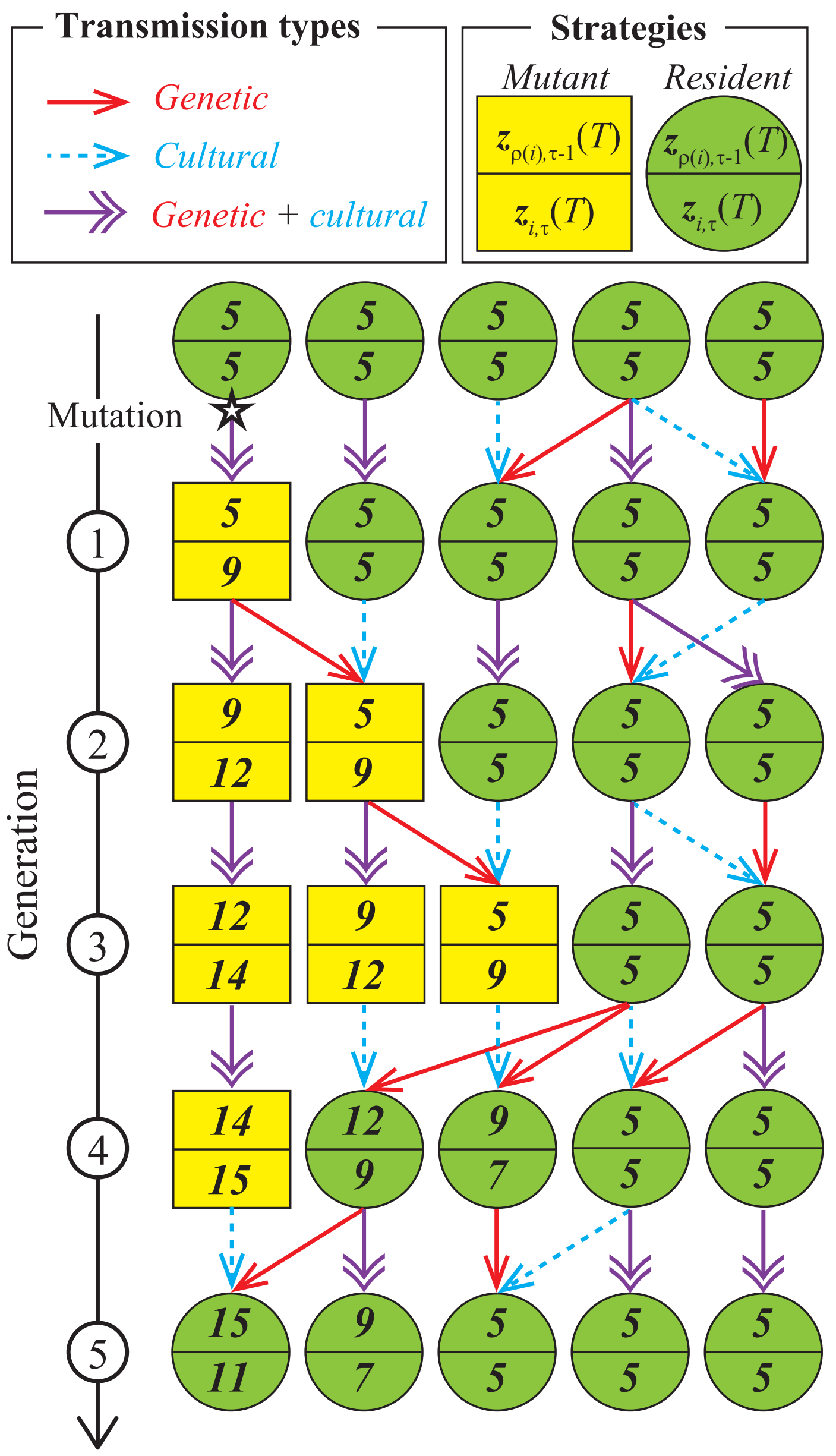


(a) $x^{*}$

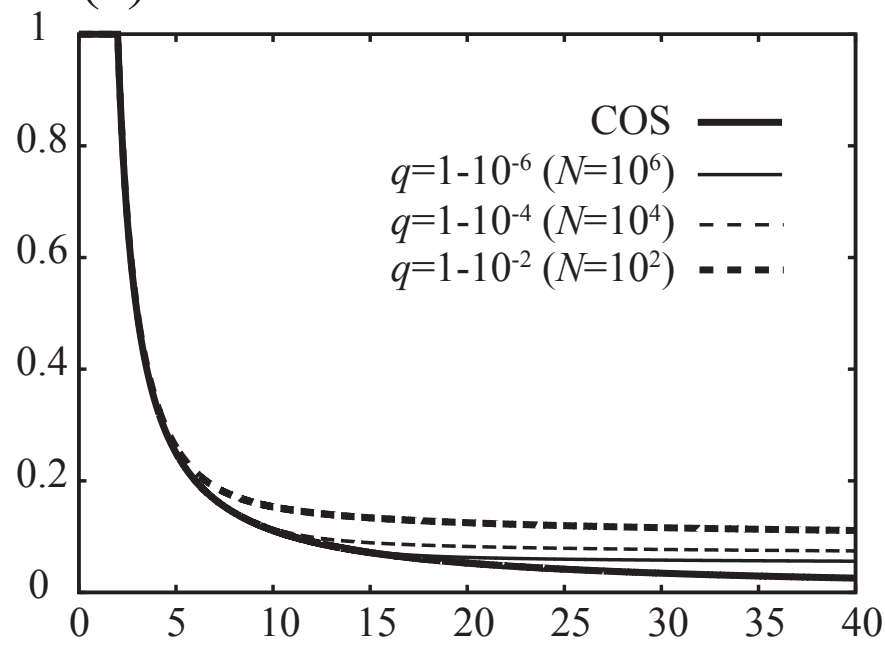

(b) $v^{*}$

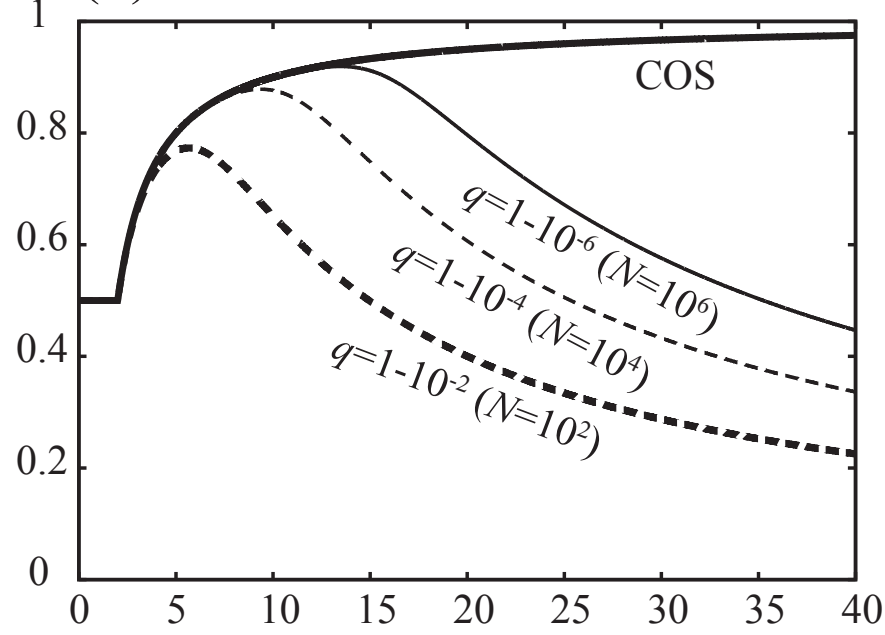

(c) $\tilde{z}^{*}(T)$

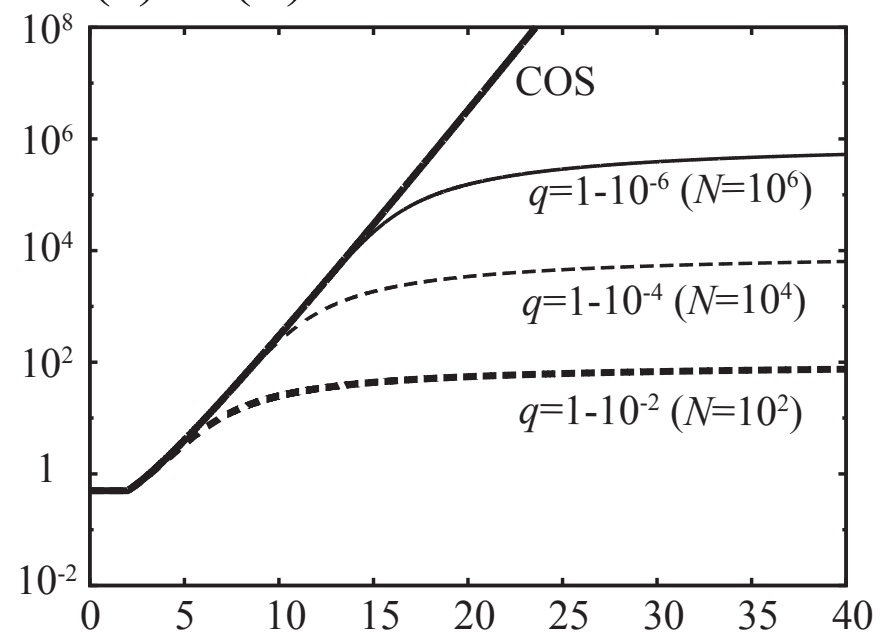

Efficiency of social learning $\beta$ 
Figure 3

(a)

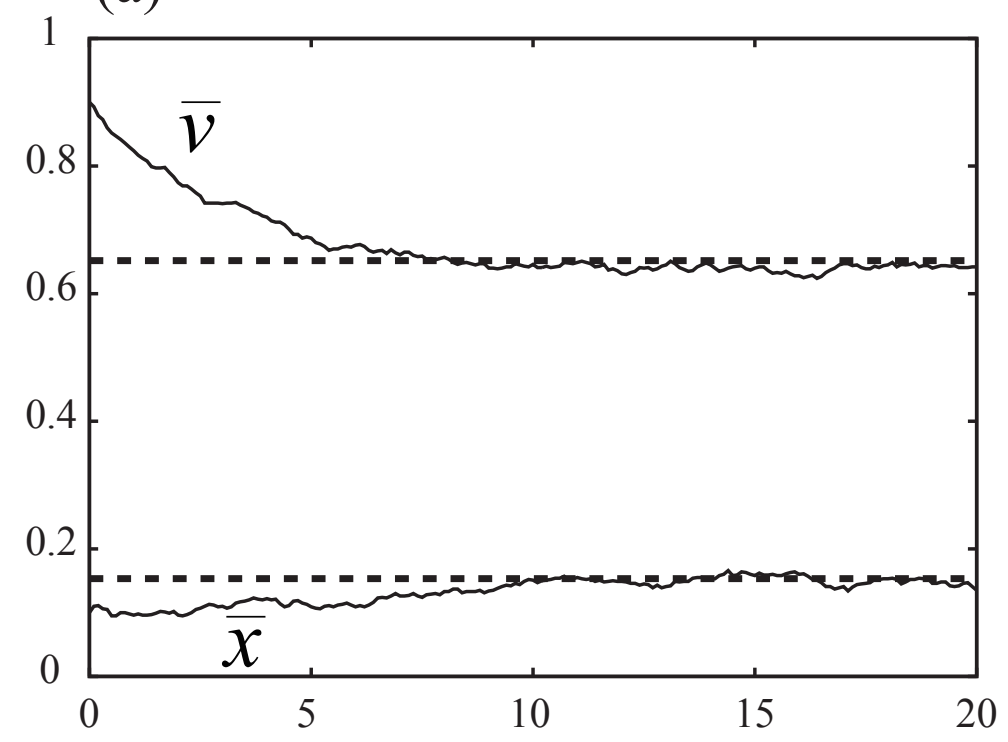

(b)

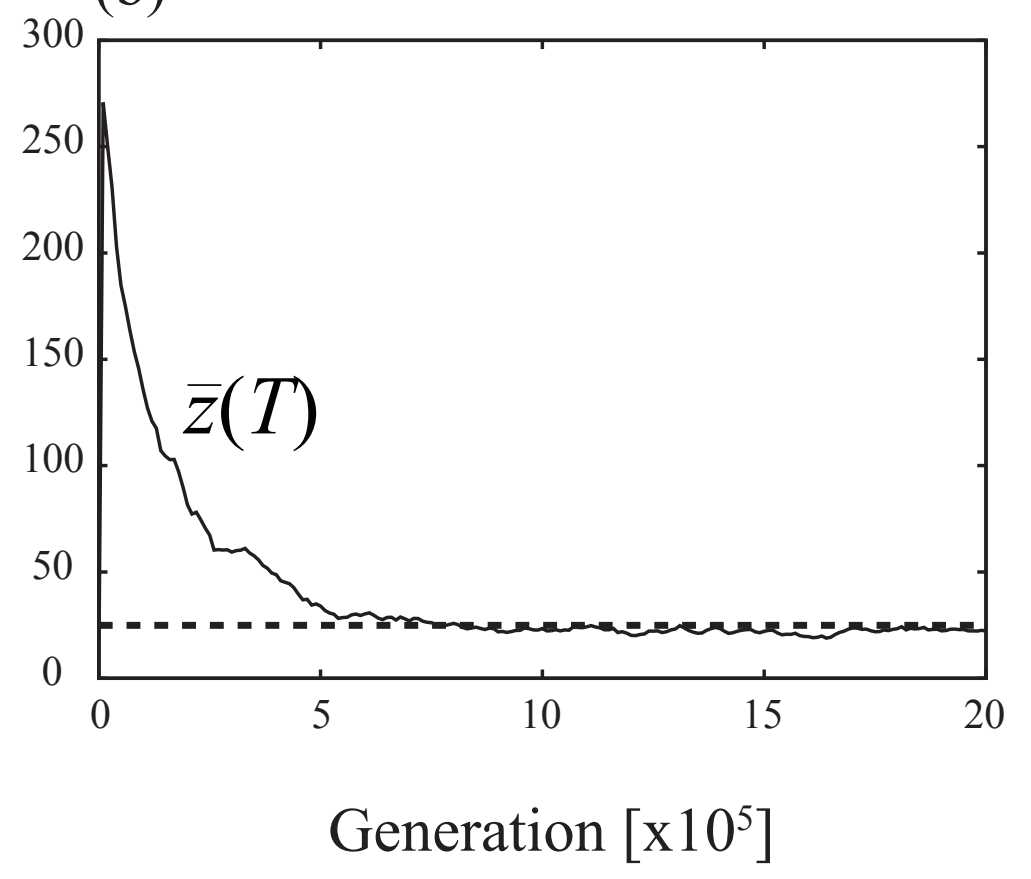






(b) $\bar{v}$
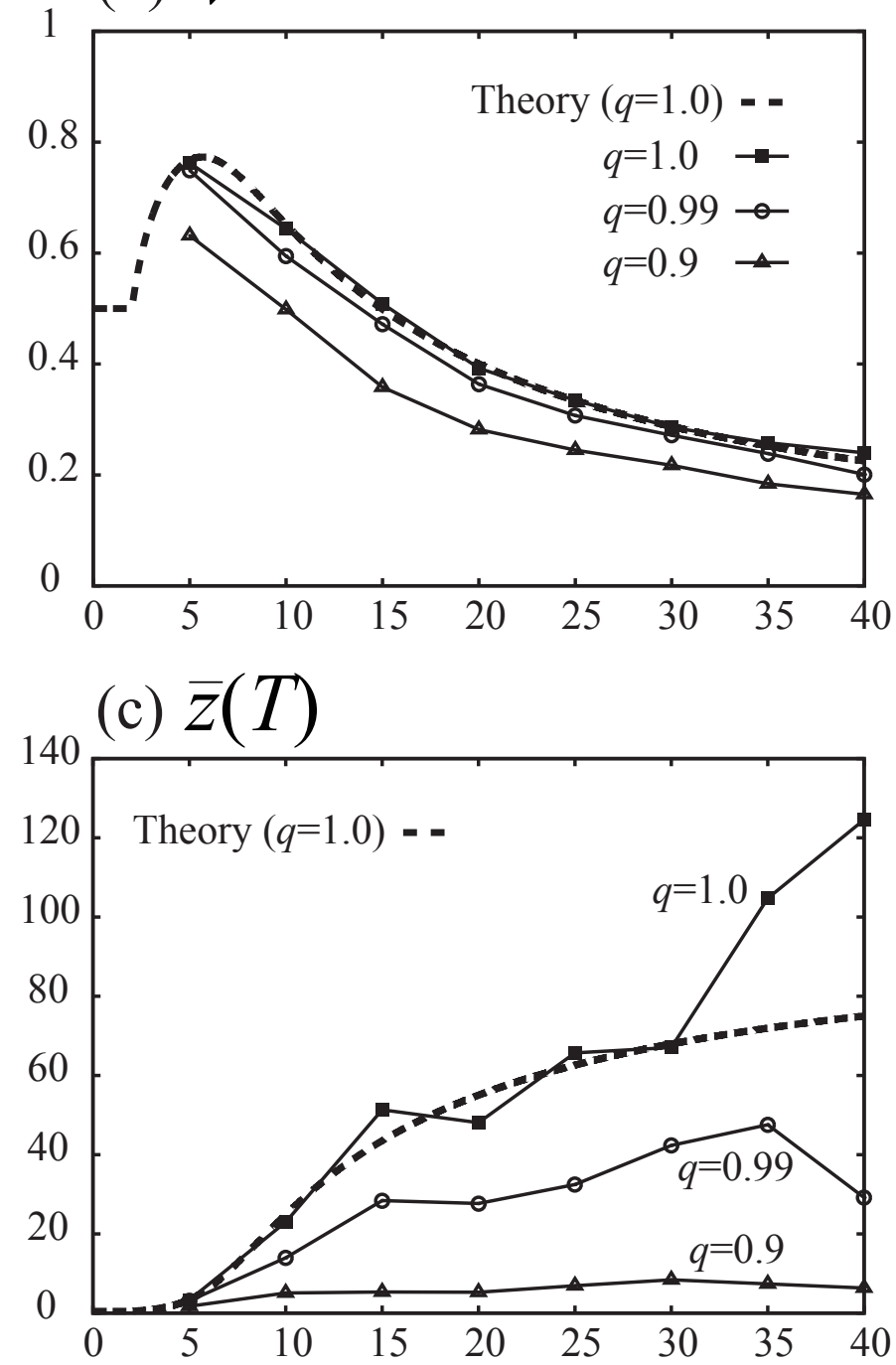

Efficiency of social learning $\beta$ 
Figure 5

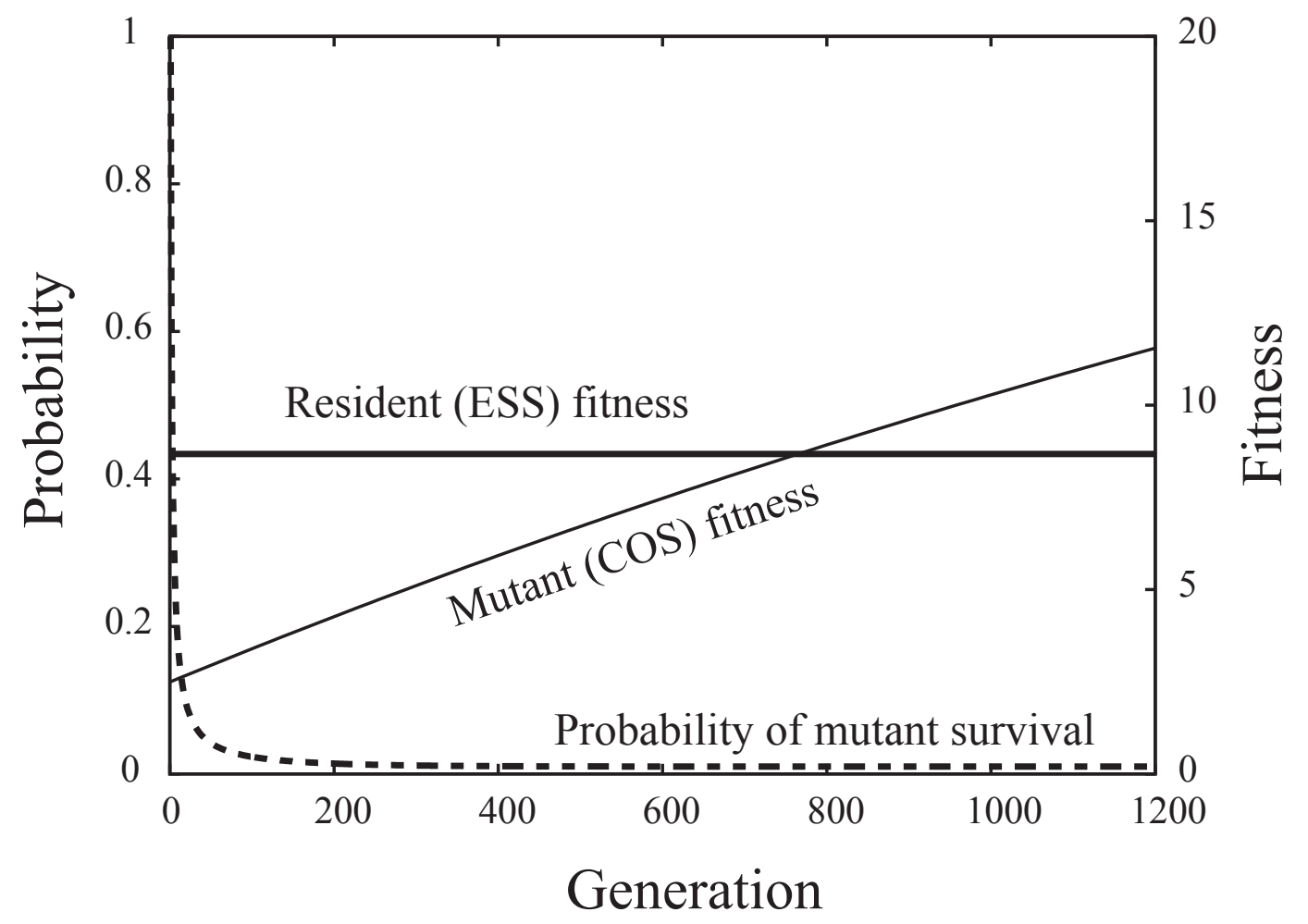




$\begin{array}{ll}N & \text { Population size } \\ q & \text { Vertical transmission rate } \\ T & \text { Lifetime } \\ \beta & \text { Efficiency of social learning } \\ \alpha & \text { Efficiency of individual learning } \\ v_{i, \tau} & \text { The fraction of the lifetime invested in learning by individual }(i, \tau) \\ & \text { The fraction of the learning time invested in individual learning by } \\ x_{i, \tau} & \text { individual }(i, \tau) \\ z_{i, \tau}(t) & \text { The } z \text {-value of individual }(i, \tau) \text { at within-generation time } t . \\ \tilde{z}(T) & \text { The equilibrium mature } z \text {-value in a genetically monomorphic population } \\ w_{i, \tau} & \text { The fitness of individual }(i, \tau) \\ v^{*}, x^{\circ}, \tilde{z}^{\circ}(T) & \text { The equilibrium fitness in a genetically monomorphic population } \\ & \end{array}$

Table 1: Notation 


\title{
Supporting information
}

\author{
A Paradox of Cumulative Culture \\ Yutaka Kobayashi $^{a, b}$, Joe Y. Wakano ${ }^{c}$, Hisashi Ohtsuki ${ }^{d}$
}

March 12, 2015

${ }^{a}$ Research Center for Social Design Engineering, Kochi University of Technology, Kochi 782-8502, Japan, ${ }^{b}$ Department of Management, Kochi University of Technology, Kochi 782-8502, Japan, ${ }^{c}$ Meiji Institute for

Advanced Study of Mathematical Sciences, Nakano, Tokyo 164-8525, Japan, ${ }^{d}$ The Graduate University for Advanced Studies, Shonan Village, Hayama, Kanagawa 240-0193, Japan

\section{Appendix A: Derivation of the COS}

2 To derive the COS, let us assume that the population is monomorphic for 3 a learning strategy $(x, v)$. Solving eq. (1) in the main text with respect to 
${ }_{4} z_{i, \tau}(t)$ under the assumption that $z_{i, \tau}(0)=0$ and $\left(x_{i, \tau}, v_{i, \tau}\right)=(x, v)$, we have

$$
z_{i, \tau}(t)=z_{\rho_{\tau}(i), \tau-1}(T)\left(1-e^{-\beta t}\right) .
$$

5 It follows that the $z$-value at the end of the social learning stage $(t=v(1-x))$

6 is given by

$$
z_{i, \tau}(v(1-x))=z_{\rho_{\tau}(i), \tau-1}(T)\left(1-e^{-\beta v(1-x)}\right)
$$

7 Further, from eq. (2) in the main text, the value of $z_{i, \tau}(t)$ at the end of the 8 individual-learning stage $(t=v)$ is given by

$$
\begin{aligned}
z_{i, \tau}(v) & =z_{i, \tau}(v(1-x))+v x \\
& =z_{\rho_{\tau}(i), \tau-1}(T)\left(1-e^{-\beta v(1-x)}\right)+v x
\end{aligned}
$$

9 Noting that $z_{i, \tau}(v)=z_{i, \tau}(T)$, we have

$$
z_{i, \tau}(T)=z_{\rho_{\tau}(i), \tau-1}(T)\left(1-e^{-\beta v(1-x)}\right)+v x
$$

10 This equation gives the between-generation dynamics of $z_{i, \tau}(T)$. From 11 eq. (14), the equilibrium value of $z_{i, \tau}(T)$, denoted by $\tilde{z}(T)$, is given by

$$
\tilde{z}(T)=\lim _{\tau \rightarrow \infty} z_{i, \tau}(T)=v x e^{\beta v(1-x)}
$$


The equilibrium fitness function, denoted by $\tilde{w}$, is therefore given by

$$
\begin{aligned}
\tilde{w}=\lim _{\tau \rightarrow \infty} w_{i, \tau} & =\lim _{\tau \rightarrow \infty} z_{i, \tau}(T) \cdot(1-v) \\
& =v(1-v) x e^{\beta v(1-x)} .
\end{aligned}
$$

The COS is the strategy $(x, v)$ which maximizes eq. (16). It is easily shown that the strategy $\left(x^{\circ}, v^{\circ}\right)$ given by eq. (6) in the main text maximizes eq. (16) and hence gives the COS.

\section{Appendix B: Derivation of the ESS in an infi-}

\section{nite population}

We define an evolutionarily stable learning strategy in an infinite population as a learning strategy that is resistant against invasion by rare mutants with any slightly deviated strategy. We will derive eq. (7) in the main text, which an ESS must satisfy.

Let $(x, v)$ and $\left(x^{\prime}, v^{\prime}\right)$ denote the resident and mutant strategies, respectively. We assume that the resident population is at cultural equilibrium, so that all residents have the $z$-value given by eq. (15) at the end of the learning stage. In order to derive the ESS, we classify individuals as follows. Residents are class 0 . The mutants who socially learned from residents are class 1 . The mutants who socially learned from class- 1 individuals are class 2 . Class- $j$ individuals are defined recursively. Note that offspring of class- $j$ mutants fall 
39 solved to yield

$$
z_{i, \tau}(T)=v^{\prime} x^{\prime} e^{\beta v^{\prime}\left(1-x^{\prime}\right)}+r^{C_{\tau}(i)}\left(v x e^{\beta v(1-x)}-v^{\prime} x^{\prime} e^{\beta v^{\prime}\left(1-x^{\prime}\right)}\right),
$$

40 where $C_{\tau}(i)$ denotes the class of individual $(i, \tau)$ and

$$
r=1-e^{-\beta v^{\prime}\left(1-x^{\prime}\right)} .
$$

${ }_{41}$ Note that eq. (18) does not depend on $i$ and $\tau$ but only on the class $C_{\tau}(i)$ of 42 individual $(i, \tau)$. This implies that the fitness of an individual also depends 
only on its class. Therefore, we let $w_{j}^{\prime}$ denote the fitness of class- $j$ mutants:

$$
w_{C_{\tau}(i)}^{\prime}:=z_{i, \tau}(T)\left(1-v^{\prime}\right), \quad\left(C_{\tau}(i) \geq 1\right)
$$

It is easily confirmed that mutants have the same fitness as residents irrespective of classes (i.e. $w_{j}^{\prime}=\tilde{w}=v(1-v) x e^{\beta x(1-v)}$ for arbitrary $j \geq 1$ ) if they adopt the same strategy as residents $\left(\left(x^{\prime}, v^{\prime}\right)=(x, v)\right)$.

Let $p_{j, \tau}$ denote the frequency of class- $j$ mutants $(j \geq 1)$ in the population in generation $\tau$. Since mutants are rare, we may assume that a mutant's role model is a mutant only when vertical transmission occurs. The offspring of a class- $j$ mutant hence belong to class- $(j+1)$ and class- 1 with probabilities $q$ and $1-q$, respectively. Further, because of rarity of mutants, the average fitness of the population is approximated by the residents' fitness $\tilde{w}$ given by eq. (16). From these arguments, it holds that

$$
p_{1, \tau+1}=\sum_{j=1}^{\infty}(1-q) \frac{w_{j}^{\prime}}{\tilde{w}} p_{j, \tau},
$$

$$
p_{j+1, \tau+1}=q \frac{w_{j}^{\prime}}{\tilde{w}} p_{j, \tau}
$$

where $j \geq 1$.

Note that the above equation is formally equivalent to the standard model of age structure. Substituting $p_{j, \tau+1}=\lambda p_{j, \tau}$ into eqs. (21) and (22) and rearranging the resulting equations, it is easily shown that the leading eigenvalue $\lambda$, i.e. the asymptotic growth rate of mutants, should satisfy the following 
${ }_{60}$ (Euler-Lotka) characteristic equation:

$$
1=\sum_{i=0}^{\infty}(1-q) q^{i} \lambda^{-i-1} \prod_{j=1}^{i+1} \frac{w_{j}^{\prime}}{\tilde{w}} .
$$

${ }_{61}$ Note that, when mutants have the same fitness as residents (i.e. $w_{j}=\tilde{w}$ 62 for all $j$ 's), $\lambda=1$ is the only solution of eq. (23). This implies that the 63 frequency of mutants remains constant when they adopt the same strategy 64 as residents.

65 Differentiating eq. (23) with respect to a mutant strategic variable $y^{\prime}$ ${ }_{66}\left(y^{\prime} \in\left\{x^{\prime}, v^{\prime}\right\}\right)$ yields

$$
\begin{aligned}
0= & \sum_{i=0}^{\infty}(1-q) q^{i}(-i-1) \lambda^{-i-2} \frac{\partial \lambda}{\partial y^{\prime}} \prod_{j=1}^{i+1} \frac{w_{j}^{\prime}}{\tilde{w}}+ \\
& \sum_{i=0}^{\infty}(1-q) q^{i} \lambda^{-i-1} \sum_{k=1}^{i+1} w_{k}^{\prime-1} \frac{\partial w_{k}^{\prime}}{\partial y^{\prime}} \prod_{j=1}^{i+1} \frac{w_{j}^{\prime}}{\tilde{w}}
\end{aligned}
$$

${ }_{67}$ Substituting $x^{\prime}=x, v^{\prime}=v, w_{j}^{\prime}=\tilde{w}$, and $\lambda=1$ into eq. (24) and rearranging ${ }_{68}$ the resulting equation yield

$$
\left.\tilde{w} \frac{\partial \lambda}{\partial y^{\prime}}\right|_{x^{\prime}=x, v^{\prime}=v}=\left.\frac{\partial w^{\prime}}{\partial y^{\prime}}\right|_{x^{\prime}=x, v^{\prime}=v},
$$

69 where

$$
w^{\prime}=\sum_{i=1}^{\infty}(1-q) q^{i-1} w_{i}^{\prime}
$$

70 If the stationary growth rate of mutants is larger than one, mutants can 71 invade. Therefore, for the resident strategy $(x, v)$ to be evolutionarily stable, 
$72 \lambda$ must be maximized at $\left(x^{\prime}, v^{\prime}\right)=(x, v)$ as a function of the mutant strategy ${ }_{73}\left(x^{\prime}, v^{\prime}\right)$. However, this and eq. (25) together imply that $w^{\prime}$ is maximized at ${ }_{74}\left(x^{\prime}, v^{\prime}\right)=(x, v)$. Thus, for our ESS analysis we may treat $w^{\prime}$ like the mutant 75 invasion fitness.

76 In fact, $w^{\prime}$ can be interpreted as the asymptotic average of the mutant 77 invasion fitness, as follows. Note that the leading eigenvector of the system 78 (21-22) is given by $\left(1, q, q^{2}, \ldots, q^{i-1}, \ldots\right)$. This means that the fraction of 79 class $i$ among mutants asymptotically approaches $(1-q) q^{i-1}$ when selection 80 is absent $\left(\left(x^{\prime}, v^{\prime}\right)=(x, v)\right)$. Thus, when selection is sufficiently weak, the 81 average fitness of mutants is asymptotically given by $\sum_{i=1}^{\infty}(1-q) q^{i-1} w_{i}^{\prime}=w^{\prime}$. $82 \quad$ Using eq. (18), (26) and (20), we find that

$$
\begin{aligned}
w^{\prime}= & \left(1-v^{\prime}\right) v^{\prime} x^{\prime} e^{\beta v^{\prime}\left(1-x^{\prime}\right)} \\
& +\left(1-v^{\prime}\right) \frac{r(1-q)}{1-r q}\left(v x e^{\beta v(1-x)}-v^{\prime} x^{\prime} e^{\beta v^{\prime}\left(1-x^{\prime}\right)}\right) .
\end{aligned}
$$

${ }_{84}\left(x^{\prime}, v^{\prime}\right)=(x, v)$. Thus, the $\operatorname{ESS}\left(x^{*}, v^{*}\right)$ satisfies

$$
\left.\frac{\partial w^{\prime}}{\partial x^{\prime}}\right|_{x^{\prime}=x=x^{*}, v^{\prime}=v=v^{*}}=0
$$

85

$$
\left.\frac{\partial w^{\prime}}{\partial v^{\prime}}\right|_{x^{\prime}=x=x^{*}, v^{\prime}=v=v^{*}}=0 .
$$

86 It is easily shown that these equations reduce to eqs. (7a) and (7b) in the 87 main text. Finally, substituting eq. (7a) in the main text into eq. (15) yields 


\section{Appendix C: Derivation of the ESS in a finite}

\section{population}

Here we derive the ESS in a finite population assuming pure vertical transmission $(q=1)$ (eq. (9) in the main text). More specifically, we show that the ESS for a finite population of size $N$ under $q=1$ is identical with the ESS for an inifinite population under $q=1-1 / N$. Thus, in terms of the ESS, decreasing the population size from $\infty$ to $N$ under $q=1$ has exactly the same effect as decreasing $q$ by $1 / N$ in an infinite population.

To compute the ESS under $q=1$, we need the fixation probability of a mutant strategy that is initially expressed by a single individual. For this purpose, we apply the method introduced by Rousset (2004) below.

Imagine that a mutant strategy $\left(x^{\prime}, v^{\prime}\right)$ is expressed by a single individual in the population of the resident strategy $(x, v)$. For convenience sake, let us reuse the classification of individuals introduced in Appendix B. Then, the initial single mutant is obviously of class 1 because there is no mutant in the previous generation. Since $q=1$ (pure vertical transmission), any mutant in any generation $\tau$ inherits culture from its own parent, which is a mutant in generation $\tau-1$. This implies that all mutants in generation $\tau$ belong to class $\tau\left(C_{\tau}(i)=\tau\right.$ for any mutant $\left.(i, \tau)\right)$, given that the mutant was introduced in generation 1. Therefore, all mutants in generation $\tau$ have equal fitnesses 
given by $w_{\tau}^{\prime}$ in eq. (20). It is important that the mutant fitness is not a stochastic variable but is determined by the number of generations passed since introduction of the initial mutant. By virtue of this property, we can treat this process as a Wright-Fisher process in which the selection coefficient depends deterministically on time (see below).

Let $P_{\tau}$ denote the frequency of mutants in generation $\tau$. Since all mutants in generation $\tau$ belong to class $\tau$, it holds that $P_{\tau}=\sum_{j} p_{j, \tau}=p_{\tau, \tau}$ in Appendix B's notation. Note that we assume a Wright-Fisher-type update for the genetic state of the population and also culture is transmitted between adjacent generations; thus, $P_{\tau}$ obeys a time-inhomogeneous Markov process with the initial state $P_{1}=1 / N$. Obviously, this stochastic process has only two absorbing states: $P_{\tau}=1$ (fixation) and $P_{\tau}=0$ (extinction). Let $\pi$ denote the fixation probability of the mutant strategy. Then, the expected frequency of mutants in the infinitely distant future should be given by

$$
\lim _{\tau \rightarrow \infty} E\left[P_{\tau}\right]=1 \cdot \pi+0 \cdot(1-\pi)=\pi
$$

where $E[\cdot]$ denotes expectation. Below we use this relationship to compute $\pi$.

Note that we can write

$$
P_{\tau}=P_{1}+\Delta P_{1}+\Delta P_{2}+\ldots+\Delta P_{\tau-1},
$$

where $\Delta P_{\tau}=P_{\tau+1}-P_{\tau}$ denotes the frequency change between generations 
${ }_{127} \tau$ and $\tau+1$ and is a stochastic variable itself. Substituting eq. (31) into eq. 128 (30) yields

$$
\begin{aligned}
\pi & =E\left[P_{1}+\sum_{\tau=1}^{\infty} \Delta P_{\tau}\right] \\
& =\frac{1}{N}+\sum_{\tau=1}^{\infty} E\left[\Delta P_{\tau}\right]
\end{aligned}
$$

129 where we used $E\left[P_{1}\right]=P_{1}=1 / N$. From the standard theory of population 130 genetics, the frequency change $\Delta P_{\tau}$ is given by

$$
\Delta P_{\tau}=\frac{w_{\tau}^{\prime}-\tilde{w}}{\tilde{w}+P_{\tau}\left(w_{\tau}^{\prime}-\tilde{w}\right)} P_{\tau}\left(1-P_{\tau}\right)
$$

131 where $\tilde{w}$ is the equilibrium fitness of residents given by eq. (16). Let us 132 define the selection coefficient $s_{\tau}$ as

$$
s_{\tau}=\frac{w_{\tau}^{\prime}-\tilde{w}}{\tilde{w}} .
$$

133 Substituting (34) into eq. (33) yields

$$
\Delta P_{\tau}=\frac{s_{\tau}}{1+P_{\tau} s_{\tau}} P_{\tau}\left(1-P_{\tau}\right) \approx s_{\tau} P_{\tau}\left(1-P_{\tau}\right),
$$

where the approximation holds for small $s_{\tau}$.

$135 \quad$ Substituting eq. (35) into eq. (32) yields

$$
\pi \approx \frac{1}{N}+\sum_{t=1}^{\infty} s_{\tau} E\left[P_{\tau}\left(1-p_{\tau}\right)\right]
$$


Note that the expectation $E\left[P_{\tau}\left(1-P_{\tau}\right)\right]$ in the above equation is itself affected by selection coefficients of up to generation $\tau-1$ (i.e., $s_{1}, s_{2}, s_{3}, \ldots, s_{\tau-1}$ ). However, Rousset (2004) has shown that the expectation $E[\cdot]$ can be approximately replaced by the expectation under neutrality (i.e. $s_{0}=s_{1}=\ldots=$ $\left.s_{t}=\ldots=0\right)$ provided selection is sufficiently weak. We denote the expectation under neutrality by $E^{\circ}[\cdot]$ following Rousset (2004). Thus, it holds that

$$
\pi \approx \frac{1}{N}+\sum_{t=1}^{\infty} s_{\tau} E^{\circ}\left[P_{\tau}\left(1-P_{\tau}\right)\right]
$$

Note that $E^{\circ}\left[2 P_{\tau}\left(1-P_{\tau}\right)\right]$ can be interpreted as the probability that two individuals drawn at random with replacement from generation $\tau$ have different genotypes under selective neutrality. Such two individuals can have different genotypes only if their ancestral lineages trace back to generation 1 without coalescing and, in addition, only one of them hits the initial mutant. From the standard coalescent theory this probability is given by

$$
\begin{aligned}
E^{\circ}\left[2 P_{\tau}\left(1-P_{\tau}\right)\right] & =\left(1-\frac{1}{N}\right)^{\tau-1} \cdot 2 P_{1}\left(1-P_{1}\right) \\
& =2 \frac{1}{N}\left(1-\frac{1}{N}\right)^{\tau},
\end{aligned}
$$

where we used $P_{1}=1 / N$.

Substituting eqs. (34) and (38) into eq. (37) yields

$$
\pi \approx \frac{1}{N}+\frac{1}{N} \sum_{\tau=1}^{\infty}\left(\frac{w_{\tau}^{\prime}}{\tilde{w}}-1\right)\left(1-\frac{1}{N}\right)^{\tau}
$$




$$
=\frac{1}{N}+\left(1-\frac{1}{N}\right)\left(\frac{w^{\prime}}{\tilde{w}}-1\right),
$$

where

$$
w^{\prime}=\sum_{\tau=1}^{\infty} w_{\tau}^{\prime} \frac{1}{N}\left(1-\frac{1}{N}\right)^{\tau-1} .
$$

Remember that for a finite population we define an ESS as the strategy that never allows a mutant strategy expressed by a single individual to have a fixation probability higher than $1 / N$ (i.e. the fixation probability of the ESS itself). This implies that for our ESS analysis we can treat $w^{\prime}$ like the mutant invasion fitness in the standard ESS analysis in an infinite-population model. Note that eq. (40) is formally identical with eq. (26) except that $q$ is replaced by $1-1 / N$. This implies that the ESS for a finite population under pure vertical transmission $(q=1)$ is equivalent with the ESS for an infinite population with $q=1-1 / N$.

\section{Appendix D: Probabilistic engagement in so- cial and individual learning}

In the main text, we assumed that social and individual learning occur in separate stages of life. In this Appendix, we instead assume that each individual engages in individual and social learning with probabilities $x$ and $1-x$, respectively, at any moment in the learning stage and derive eq. (14) under some additional assumptions. Thus, the results of the present paper 
Integrating both equations yield

182 Likewise, $z_{i, \tau, I L}(t)$ follows

$$
\frac{d}{d t} z_{i, \tau, S L}(t)=\beta(1-x)\left(z_{\rho_{\tau}(i), \tau-1}(T)-z_{i, \tau, S L}(t)\right) . \quad(0 \leq t \leq v)
$$

$$
\frac{d}{d t} z_{i, \tau, I L}(t)=\alpha x=x . \quad(0 \leq t \leq v)
$$

$$
z_{i, \tau, S L}(v)=z_{\rho_{\tau}(i), \tau-1}(T)\left(1-e^{-\beta v(1-x)}\right) .
$$




$$
z_{i, \tau, I L}(v)=x v .
$$

184 Summing up eqs. (43) and (44) yields

$$
\begin{aligned}
z_{i, \tau}(v) & =z_{i, \tau, S L}(v)+z_{i, \tau, I L}(v) \\
& =z_{\rho_{\tau}(i), \tau-1}(T)\left(1-e^{-\beta v(1-x)}\right)+x v
\end{aligned}
$$

Since knowledge does not grow in the exploitation stage, we have $z_{i, \tau}(T)=$ $z_{i, \tau}(v)$. Substituting this into eq. (45) yields eq. (14).

Note that the above derivation requires to specify the interpretation of the $z$-value and the structure of knowledge. On the other hand, the separatestep framework of the original model incorporates a wider class of knowledge structure and allows more general interpretations of the results in this respect.

\section{Literature Cited}

- Rousset, F. 2004. Genetic structure and selection in subdivided populations. Princeton University Press. 